\title{
Escalas de avaliação para Terapia Ocupacional no Brasil
}

\section{Evaluation scales for Occupational Therapy in Brazil}

\author{
Giseli de Fátima dos Santos Chaves, Alexandra Martini de \\ Oliveira $^{2}$, Orestes Vicente Forlenza ${ }^{3}$, Paula Villela Nunes ${ }^{4}$
}

CHAVES, G. F. S.; OLIVEIRA, A. M.; FORLENZA, O. V.; NUNES, P. V. Escalas de avaliação para Terapia Ocupacional no Brasil. Rev. Ter. Ocup. Univ. São Paulo, v. 21, n. 3, p. 240-246, set./dez. 2010.

RESUMO: A produção científica relativa às intervenções e avaliações em Terapia Ocupacional têm avançado. Instrumentos de avaliação são cada vez mais utilizados para avaliar resultados de intervenções nos Estados Unidos, Canadá, Austrália e em países da Europa. No Brasil, entretanto, instrumentos traduzidos e validados ainda são pouco utilizados e difundidos entre terapeutas ocupacionais. Diante disso, o objetivo desta revisão de literatura é conhecer os instrumentos e escalas de avaliação em Terapia Ocupacional que estão validados para língua portuguesa e disponíveis para o uso no Brasil. Para a pesquisa foi utilizada a base de dados da Biblioteca Virtual em Saúde (BVS) a partir de 1969 e trabalhos disponíveis na biblioteca do Serviço de Terapia Ocupacional do Instituto de Psiquiatria da Faculdade de Medicina da Universidade de São Paulo. Apenas sete instrumentos de avaliação foram encontrados, um indicativo de que no Brasil há poucos instrumentos validados para o uso em Terapia Ocupacional. O uso de instrumentos de avaliação possibilita o estabelecimento de objetivos terapêuticos e a mensuração dos resultados obtidos em terapia, sendo muito importantes para o reconhecimento clínico e científico da Terapia Ocupacional, além de possibilitar a produção de conhecimento específico na área. Desta forma, se faz necessário o aumento e ampliação da discussão das formas de sistematização das avaliações em Terapia Ocupacional no Brasil.

DESCRITORES: Avaliação; Estudos de validação; Literatura de revisão como assunto; Terapia ocupacional.

\footnotetext{
1. Terapeuta ocupacional, colaboradora do Ambulatório de Psicogeriatria do LIM-27 e do Hospital-Dia de Idosos do Instituto de Psiquiatria do Hospital das Clínicas da Faculdade de Medicina da Universidade de São Paulo (IPq-HCFMUSP).

2. Terapeuta ocupacional, Mestre em Psiquiatria pelo IPq-HCFMUSP, colaboradora do Ambulatório de Psicogeriatria do LIM 27, do Hospital-Dia de Idosos do IPq-HCFMUSP e Diretora do Serviço de Terapia Ocupacional do IPq-HCFMUSP.

3. Médico psiquiatra, Professor Associado, Livre-Docente, pelo Departamento de Psiquiatria da FMUSP, Vice-diretor do Laboratório de Neurociências LIM-27, IPq-HCFMUSP .

4. Médica psiquiatra, Doutora em Psiquiatria pelo IPq-HC-FMUSP. Médica Pesquisadora do LIM-27 e coordenadora do Hospital-Dia de Idosos - IPq-HCFMUSP.

Endereço para correspondência: Departamento de Fisioterapia, Fonoaudiologia e Terapia Ocupacional da FMUSP. Rua Cipotânea, 51, Cidade Universitária, São Paulo, SP. CEP 05360-160.
} 


\section{INTRODUÇÃO}

$\mathrm{D}$ esde o surgimento da Terapia Ocupacional, no início do século XX, muitos métodos de avaliação sistemática vêm sendo desenvolvidos por terapeutas ocupacionais da Europa, Estados Unidos, Canadá e Austrália. Estes métodos precisam ser padronizados, confiáveis, válidos e sensíveis a mudanças clínicas para que se possam avaliar resultados (na prática clínica e em pesquisas científicas) e comprovar o custo-benefício das intervenções (MAGALHÃES, 1997; TEDESCO, 2002).

Para a elaboração dos testes, o processo de construção é longo e dispendioso e engloba: 1. Planejamento: onde é definido o objetivo e tipo de população alvo do teste; 2 . Construção: os itens são desenvolvidos, delineando a versão piloto; 3 . Avaliação quantitativa: a versão piloto passa por uma revisão geral, tem sua confiabilidade testada até alcançar o formato final; 4. Validação: o instrumento é aplicado em um número significativo da população. Os dados resultantes são coletados para derivação de normas e para o exame das validades concorrente, de critério e de construto (MAGALHÃES et al., 2004).

O estudo da confiabilidade é feito através da comparação de diversas aplicações do instrumento ao mesmo indivíduo. Comumente, a avaliação é feita em duas etapas: confiabilidade teste/re-teste e entre dois entrevistadores. A avaliação da confiabilidade teste/re-teste é realizada através da aplicação do instrumento, por um mesmo entrevistador, em dois momentos distintos $\left(t_{0}\right.$ e $\left.t_{1}\right)$, dentro de um período pré-estabelecido. Os resultados da primeira etapa $\left(\mathrm{t}_{0}\right)$ são comparados com os resultados da segunda etapa $\left(t_{1}\right)$ (MENEZES, 1998; TEDESCO, 2000).

A avaliação da confiabilidade entre dois entrevistadores é testada através da aplicação do instrumento, por dois entrevistadores diferentes $\left(\mathrm{E}_{1}\right.$ e $\left.\mathrm{E}_{2}\right)$, em um mesmo indivíduo. É importante que estas duas aplicações sejam realizadas separadamente, para eliminar um possível grau de concordância causado pela observação conjunta dos resultados (MENEZES, 1998). O grau de concordância entre ambas as avaliações é medido por um coeficiente de confiabilidade, e existem várias formas de calculá-lo. A escolha entre as diversas formas dependerá da natureza do instrumento que se deseja avaliar (BARTKO, 1991).

A validade de um instrumento pode ser definida como sua capacidade em realmente medir aquilo que ele se propõe a medir (OLIVEIRA, 1995). Ela envolve um componente conceitual e um componente operacional. O primeiro diz respeito ao julgamento do investigador de se o instrumento mede o que deveria medir. Esse julgamento é subjetivo, não sendo possível avaliar esse aspecto com métodos estatísticos (ALMEIDA FILHO, 1989). Já a validade operacional envolve uma avaliação sistemática do instrumento. Para a comparação geralmente são utilizadas análises estatísticas (MENEZES, 1998; TEDESCO, 2002).

Três aspectos de validade operacional podem ser avaliados: 1. Validade de conteúdo: é o estudo da abrangência do instrumento nos diferentes aspectos do seu objeto; 2. Validade de critério: avalia-se o grau com que $o$ instrumento discrimina entre pessoas que diferem em determinadas características de acordo com um critério padrão. Quando o instrumento e o critério são aplicados simultaneamente, fala-se de validade concorrente; quando o critério é avaliado no futuro, fala-se de validade preditiva. 3. Validade de construto: demonstra que $o$ instrumento realmente mede aquilo a que ele se propõe medir (OLIVEIRA, 1995; MENEZES, 1998; TEDESCO, 2002). A validade divergente ou discriminante é usada para substanciar a identidade dos constructos que elas exploram. Ela ocorre quando os escores que devem diferir se correlacionam de maneira baixa. A validade convergente ocorre quando diferentes escores definidos para avaliar o mesmo constructo possuem correlações altas, demonstrando a semelhança dos constructos avaliados (URBINA, 2007).

Para a utilização de instrumentos desenvolvidos em outros países, além de ser necessária a realização da tradução transcultural, o estudo da confiabilidade e de validade precisa ser refeito nesta nova versão devidamente traduzida.

A tradução transcultural procura tornar o instrumento traduzido o mais próximo possível da sua versão original. Para isso, diversos aspectos são estudados: Equivalência conceitual: refere-se à equivalência do conceito da cultura original em comparação à cultura-alvo; Equivalência de itens: indica se os itens que compõem o instrumento estimam os mesmos domínios e se são relevantes nas duas culturas; Equivalência semântica: consiste na tradução do instrumento original, buscando conservar o significado das palavras e para atingir o mesmo efeito nas duas culturas distintas; Equivalência operacional: preservação das medidas operacionais empregadas antes e durante da aplicação da escala original, e Equivalência e mensuração: refere-se às propriedades psicométricas utilizadas para testar a equivalência de um instrumento em duas línguas diferentes, avaliada por meio de medidas de confiabilidade e validade (PESCE, 2005).

De acordo com Magalhães (1997), no Brasil, ainda é pequeno o número de instrumentos padronizados, válidos e fidedignos específicos para Terapia Ocupacional. Tal 
carência de instrumentos de avaliação dificulta a prática de coleta de dados para demonstração de resultados obtidos em terapia.

Atualmente, a importância de embasamento científico nas intervenções e avaliações e a necessidade de ampliação da ciência da Terapia Ocupacional vêm sendo amplamente discutidas (TICKLE-DEGNEN, 2000; TSE; PENMAN, 2000).

Desta forma, esta revisão da literatura busca mapear e conhecer os instrumentos e escalas de avaliação validados para a língua portuguesa, preferencialmente no Brasil, a fim de ampliar seus usos na prática clínica e em pesquisas científicas.

\section{MÉTODOS}

Instrumentos de avaliação específicos de Terapia Ocupacional foram pesquisados na biblioteca do Serviço de Terapia Ocupacional do Instituto de Psiquiatria da Faculdade de Medicina da Universidade de São Paulo e na base de dados indexada da Biblioteca Virtual em Saúde (BVS) (a qual inclui Pubmed, Lilacs e Scielo) de 1969 a 2010. Na pesquisa na BVS foram utilizadas as palavras "terapia ocupacional" e "validação", cujo resultado foi de 12 trabalhos, refinados em 7, de acordo com o assunto. A pesquisa com as palavras "terapia ocupacional" e "validade", encontrou 237 trabalhos, cuja refinação decorreu nos mesmos 7 artigos da pesquisa anterior. Os instrumentos poderiam ser procedentes das diversas abordagens teóricas de Terapia Ocupacional utilizadas mundialmente, porém, deveriam ter seu processo de validação e/ou elaboração estabelecidos em paises de língua portuguesa, preferencialmente no Brasil. Foram considerados os artigos escritos em inglês e português, além de livros, dissertações de mestrado e teses de doutorado realizadas no Brasil.

\section{RESULTADOS}

Foram encontradas poucas escalas de avaliação, específicas da Terapia Ocupacional, que tenham sido elaboradas ou devidamente adaptadas e validadas para o uso no Brasil. Os instrumentos encontrados na literatura até o momento são:

- Entrevista da História do Desempenho Ocupacional (EHDO) (BENETTON; LANCMAN, 1998) é uma entrevista semi-estruturada formada por duas partes. A primeira parte consiste na própria entrevista, em que é avaliado o desempenho ocupacional do individuo no passado e presente de acordo com os seguintes itens: organização das rotinas da vida diária; papéis de vida; interesses, valores e metas; percepção das habilidades e responsabilidades e influências ambientais. A segunda parte consiste em um formulário padrão para reportar os resultados da entrevista (pontuação) e a narrativa de história de vida, feita a partir de questões abertas relacionadas aos cinco itens já citados. A versão original em inglês feita em 1989 foi revisada em 1998, por falha na demonstração de suas qualidades psicométricas e pela entrevista não ser compatível para a utilização de terapeutas ocupacionais com outro referencial teórico que não o Modelo de Ocupação Humana idealizado por Kielhofner em 1985. Portanto, a versão validada em português foi feita a partir da versão em inglês não revisada-

- Escala de Observação Interativa de Terapia Ocupacional - EOITO (OLIVEIRA, 1995), mede mudanças em pacientes durante o período de sessões de Terapia Ocupacional. É uma escala composta por 16 itens (cuidado pessoal, execução de atividades, demonstração de interesse, comunicação verbal, interação social, referência a fatos irreais, alucinações, orientação, psicomotricidade aumentada, linguagem acelerada, irritabilidade, aceitação de limites, expressão de auto-estima e comportamento inabitual). Apresenta três graduações, indicando uma ordem crescente da gravidade nas manifestações psicopatológicas. A avaliação consiste não apenas na observação, mas na interação entre avaliador e paciente. Pode ser usada em enfermarias, hospitais-dia e ambulatórios de Saúde Mental. Porém, seu uso é limitado por avaliar apenas sintomas psiquiátricos.

- Auto-Avaliação do Funcionamento Ocupacional - SAOF (TEDESCO, 2000; 2010) baseada no Modelo de Ocupação Humana (KIELHOFNER, 1985), se trata de um instrumento estruturado que originalmente possui uma versão longa, uma simplificada e outra para crianças de 10 a 13 anos. A versão longa e a simplificada podem ser utilizadas em todos os tipos de acometimentos e em clientes com idade entre 14 e 85 anos. É formada por 23 questões que cobrem 7 áreas de conteúdo: a causalidade pessoal se refere a imagem e as expectativas que o sujeito faz de si próprio; valores que organizam o comportamento do sujeito através de suas prioridades; interesses são atividades que o individuo gosta e tem prazer em realizar; papéis são o conjunto de características no comportamento que são esperadas para a idade e posição social específica; hábitos estruturam as ações diárias para conseguir maior eficiência no desempenho de tarefas exigidas pelo seu papel ocupacional; habilidades (físicas ou mentais) auxiliam na expressão, no contato social e na ação; meio-ambiente diz respeito à variedade de locais onde a pessoa passa o seu 
tempo e inclui as pessoas com que se relaciona, objetos e recursos sociais. Para avaliar a percepção individual do paciente sobre estes itens é utilizada uma escala de 3 pontos de classificação (ponto forte, adequada e necessidade de melhora). Desta forma, a SAOF prioriza o estímulo ao engajamento do paciente no tratamento. No entanto, em um estudo (BEZERRA, 2006) sobre a percepção do funcionamento ocupacional e da qualidade de vida de pacientes que faziam hemodiálise em que a SAOF foi utilizada como instrumento de avaliação, parte considerável dos pacientes teve dificuldade em entender alguns de seus tópicos. Apesar disto, a SAOF foi bastante útil na coleta de informações. Desta forma, mais estudos são necessários para avaliar sua utilidade na mensuração dos resultados de intervenções em Terapia Ocupacional.

- Classificação de idosos quanto à capacidade para o autocuidado - (CICAC) (ALMEIDA, 2003) possui duas versões: simplificada e grupal. A grupal é mais longa e o nome foi dado por ter sido ajustada através da contribuição de 15 terapeutas ocupacionais. A versão simplificada constitui-se de 20 questões e a versão grupal, 22 questões. Estas se referem aos seguintes itens: Arranjo Doméstico e Familiar e sua Potencial Rede de Suporte; Perfil Social; Universo Ocupacional e Capacidade Funcional. No item Capacidade Funcional, são avaliadas as Atividades de Vida Diária (básicas e instrumentais), Lazer e Trabalho (remunerado e não-remunerado). Sua validade e confiabilidade foram estabelecidas nas duas versões. $\mathrm{O}$ instrumento é bem abrangente e admite a complexidade do sujeito a ser avaliado, porém, mesmo a versão simplificada, exige um tempo longo para sua aplicação. Além disso, ainda não existem estudos avaliando a sua sensibilidade às mudanças ao longo do tempo, como, por exemplo, no caso de uma reavaliação.

- Avaliação da Coordenação e Destreza Motora (ACCORDEM) (MAGALHÃES et al., 2004) teste para detecção de transtorno da coordenação motora em crianças de 4 a 8 anos de idade. Os itens de observação direta (áreas de Coordenação e Destreza Manual e de Coordenação Corporal e Planejamento Motor) têm escore numérico, baseado no tempo e acuidade da resposta. Os questionários de pais e professores (Desempenho Funcional em casa e na escola) são pontuados em escala de quatro pontos, indicando a freqüência dos comportamentos observados (nunca/raramente, ocasionalmente, freqüentemente e sempre). A avaliação ocorre num contexto de brincadeira, visando facilitar a aceitação pelas crianças. AACOORDEM versão 1.0 é uma escala bastante extensa, que foi formatada no Brasil, levando em consideração muitas outras escalas de desenvolvimento infantil.. Seu processo de validação e confiabilidade foi estudado em 2009 (CARDOSO; MAGALHÃES, 2009).

- Lista de Identificação de Papéis Ocupacionais (CORDEIRO, 2005) baseado em conceitos descritos no Modelo de Ocupação Humana (KIELHOFNER, 1985). É auto-aplicável e sua realização constitui-se de duas etapas. Na primeira etapa, o cliente define sua participação ou não em papéis ocupacionais no passado, presente e futuro. Esses papéis são pré-estabelecidos pela avaliação (estudante, trabalhador, voluntário, cuidador, serviço doméstico, amigo, membro de família, religioso, passatempo/amador, participantes em organizações e outros para identificar papéis não listados). Na segunda etapa, o cliente atribui a cada papel descrito (desempenhado e não-desempenhado) seu grau de importância, podendo variar de nenhuma importância, alguma importância a muita importância. Pode ser utilizado em adolescentes, adultos e idosos, com qualquer tipo de disfunção e exige tempo curto para sua administração. Mais estudos são necessários para avaliar sua sensibilidade a mudanças, no caso de reavaliação, e verificação de resultados de intervenção.

- Loewenstein Occupational Therapy Cognitive Assessment_(LOTCA) (GAMEIRO; FERREIRA, 2006) é uma bateria de testes elaborada para avaliar as capacidades cognitivas básicas de pacientes jovens e adultos com alguma lesão cerebral (traumatismos, tumores, acidentes vasculares encefálicos). É baseada em teorias neuropsicológicas e cognitivas de processamento de informação. As funções cognitivas avaliadas pelos testes incluem a orientação, percepção visual e espacial, práxis motora, organização visuomotora e operações de pensamento. O LOTCA objetiva fornecer um perfil cognitivo, por isso, não há cálculo das pontuações totais. As pontuações referemse às funções separadas (orientação, percepção visual e espacial, práxis motora, organização visuomotora e operações de pensamento) e são usadas para avaliar em qual destas funções há um déficit que precisa de intervenção terapêutica. Por exemplo, um paciente pode ter um prejuízo na organização motora e estar bem orientado. Vale notar que o LOTCA foi validado apenas em Portugal, no entanto, vêm sendo usado no Brasil.

\section{DISCUSSÃO}

Na literatura internacional, especialmente em países como Estados Unidos, Austrália e Canadá, a produção científica para a elaboração e utilização de instrumentos de avaliação em Terapia Ocupacional tem sido grande (MAGALHÃES, 1997). Há uma grande diversidade de referenciais teóricos e de usos para determinadas 
CHAVES, G. F. S. et al. Escalas de avaliação para Terapia. Rev. Ter. Ocup. Univ. São Paulo, v. 21, n. 3, p. 240-246, set./dez. 2010.

populações, entre outros aspectos. Na revisão da literatura internacional realizada por Tedesco, 2000, que procurou apenas por instrumentos padronizados de avaliação de Terapia Ocupacional em Saúde Mental, foram encontrados 16 instrumentos, incluindo o instrumento que foi validado em seu trabalho, a SAOF. Na pesquisa realizada para esta revisão na base de dados BVS - Biblioteca Virtual em Saúde, utilizando os termos "validade" e "terapia ocupacional", foram encontrados 237 artigos, sendo 95 relacionados com a validação, confiabilidade, utilidade clínica e/ou elaboração de instrumentos de avaliação de Terapia Ocupacional internacionais.

Um fator que contribui para esta produção é a crescente preocupação em estruturar intervenções e avaliações em bases científicas e ampliar a ciência da Terapia Ocupacional. Além disso, o uso destes instrumentos se faz mais freqüente pela necessidade econômica dos serviços de saúde em justificar o custo-benefício de intervenções e avaliar resultados obtidos, pressionados em parte pelo sistema econômico e de assistência de saúde existente (MAGALHÃES, 1997).

Por outro lado, como pode-se constatar, existem poucas escalas de avaliação em Terapia Ocupacional que podem ser utilizadas no Brasil. Apenas sete instrumentos traduzidos para a língua portuguesa e validados foram encontrados nesta revisão da literatura. Dentre os instrumentos validados para o português, alguns são específicos para algumas faixas etárias (crianças ou idosos) ou doenças (psiquiátricas ou neurológicas), outros são mais abrangentes e avaliam diversas áreas do desempenho ocupacional.

As escalas já validadas e/ou elaboradas em português encontradas avaliam o desempenho ocupacional sob diferentes aspectos. Na CICAc, o desempenho ocupacional é investigado nas atividades de vida diária, trabalho e lazer. Na ACCORDEM ele é investigado na produtividade (no papel de estudante e no brincar) e no auto-cuidado. A EOITO avalia principalmente componentes cognitivos, psicológicos, afetivos e sociais durante a realização de uma atividade, mas avalia também o auto-cuidado (higiene) e o lazer (sociabilidade). Já a LOTCA avalia apenas os componentes cognitivos por meio de desempenho ocupacional e ainda não foi traduzida e validada para o português do Brasil. A investigação dos componentes (cognitivos, psicológicos, físicos, etc.) do desempenho ocupacional é muito importante, no entanto, é necessária a correlação destes componentes com as atividades que o sujeito precisa realizar em seu cotidiano e nas quais apresenta dificuldades.

A ACCORDEM, assim como a CICAc, abrangem apenas determinados estágios de desenvolvimento (crianças e idosos, respectivamente). A ACCORDEM, EOITO; LOTCA são escalas desenvolvidas para avaliar determinadas condições clínicas (atraso no desenvolvimento neuropsicomotor, sintomas psicopatológicos e déficits cognitivos, respectivamente). Por causa desta especificidade, há uma limitação do uso destas escalas na prática.

Já a EHDO, a SAOF e a Lista de Identificação de Papéis Ocupacionais avaliam o comportamento ocupacional e os papéis ocupacionais desempenhados pelo individuo, o que compreende todas as atividades realizadas ou que precisam ser realizadas pelo individuo em seu cotidiano. Entretanto, o terapeuta ocupacional, que as utilize em sua prática, necessita conhecer a abordagem teórica do Modelo de Ocupação Humana - MOH (KIELHOFNER, 1985), que fundamenta estas avaliações. Pode-se observar isso em um estudo em que foi utilizada a SAOF como medida de avaliação (BEZERRA, 2006). Os pacientes participantes desta pesquisa relataram dificuldades em entender conceitos presentes no instrumento, os quais eram próprios deste modelo teórico. AACCORDEM, EOITO, CICAc e LOTCA não possuem fundamentação em uma abordagem teórica específica de Terapia Ocupacional.

A EHDO, composta por questões abertas e fechadas, também possibilita ao individuo detalhar seu desempenho e relatar queixas individuais. Já a SAOF, Lista de Identificação de Papéis Ocupacionais, ACCORDEM, EOITO, CICAc e LOTCA são instrumentos que diminuem esta possibilidade, pois se tratam de questionários estruturados com respostas pré-estabelecidas a serem assinaladas pelo individuo.

Deve-se, além de observar todos estes aspectos discutidos, considerar o tempo de administração para a escolha de um instrumento. A EHDO, CICAc e ACCORDEM são avaliações que demandam muito tempo para suas aplicações. A SAOF demanda pouco tempo, porém, algumas questões são complexas e podem necessitar de esclarecimento, acarretando em um aumento do tempo de administração. A Lista de Identificação de Papéis Ocupacionais, EOITO e LOTCA, são de rápida aplicação. A EOITO e LOTCA podem até ser aplicadas durante a intervenção.

Diante desta lista de instrumentos de avaliação e suas principais características, é possível refletir e determinar qual destes instrumentos será mais adequado ao uso no exercício de cada terapeuta ocupacional, seja na prática clínica ou em pesquisas cientificas. Com a sistematização das avaliações, se pode obter dados clínicos, delinear objetivos, direcionar intervenções e evidenciar mudanças clínicas na população atendida. Desta forma, a comprovação de resultados obtidos durante as intervenções de Terapia Ocupacional com estes instrumentos justificam a atuação do profissional 
terapeuta ocupacional e aumentam a produção científica da profissão.

\section{CONCLUSÃO}

Esta revisão demonstra a necessidade do aprofundamento da discussão da sistematização dos métodos de avaliação específicos de Terapia Ocupacional. No Brasil, ao contrário de outros países, existem poucas avaliações válidas e fidedignas específicas de Terapia Ocupacional.

Algumas delas são adequadas apenas para alguns tipos de população, condições clínicas ou faixas etárias. Algumas avaliam apenas os componentes do desempenho ocupacional, enquanto outras avaliam as áreas do desempenho ocupacional. Há instrumentos em que são baseados em teorias específicas de Terapia Ocupacional. Algumas são mais rápidas em sua aplicação e outras incorporam elementos qualitativos, além dos quantitativos em suas estruturas. Porém, a utilização e conhecimento destes instrumentos ainda são limitados.

Este quadro dificulta a comprovação da eficácia de muitas das intervenções em Terapia Ocupacional, por meio destes instrumentos. A necessidade de sistematizar avaliações, abrangendo a coleta de dados, o estabelecimento de objetivos terapêuticos e a mensuração dos resultados obtidos em terapia, precisa ser considerada e estimulada. Já que favorece o reconhecimento clínico e científico da profissão, além de possibilitar a produção de conhecimento específico da área e a confiabilidade das intervenções.

CHAVES, G. F. S.; OLIVEIRA, A. M.; FORLENZA, O. V.; NUNES, P. V. Evaluation scales for Occupational Therapy in Brazil. Rev. Ter. Ocup. Univ. São Paulo, v. 21, n. 3, p. 240-246, set./ dez. 2010

\begin{abstract}
S: The scientific literature of interventions and evaluations in Occupational Therapy has advanced. Assessment instruments are increasingly used to evaluate outcomes of interventions in the U.S.A., Canada, Australia and European countries. However, in Brazil, instruments translated and validated are not much used and known among occupational therapists. Therefore, the objective of this review of the literature is to know which instruments and rating scales in Occupational Therapy are validated for portuguese language and available for use in Brazil. The database of the Virtual Health Library (BVS) from 1969 was used as well as studies available in the library of the Occupational Therapy Service of the Psychiatry Institute, University of Sao Paulo. Only seven assessments instruments were found what denotes there are few validated instruments for use in Occupational Therapy in Brazil. The use of assessment tools allows the establishment of therapeutic goals and measurement of results in therapy which are very important for the clinical and scientific recognition of the Occupational Therapy and through this is possible the production of specific knowledge in the area. Thus, it is necessary to increase and expand of the discussion of the systematic ways to evaluate in Occupational Therapy in Brazil.
\end{abstract}

KEY WORDS: Evaluation; Validation studies; Review literature as topic; Occupational therapy.

\title{
REFERÊNCIAS
}

ALMEIDA, M. H. M. Validação do instrumento CICAc: Classificação de idosos quanto a capacidade para o autocuidado. Tese (Doutorado em Saúde Pública). São Paulo: Faculdade de Saúde Pública da Universidade de São Paulo, 2003.

ALMEIDA FILHO, N. (Org.). Epidemiologia sem números: uma introdução crítica à ciência epidemiológica. Rio de Janeiro: Editora Campus, 1989.

BARTKO, J. J. Measurement and Reliability: Statistical Thinking
Considerations. Schizophrenia Bull.; v. 17, p. 483-489. 1991.

BENETTON, M. J. ; LANCMAN, S. Estudo de confiabilidade e validação da "Entrevista da história do desempenho ocupacional". Rev. Ter. Ocup. Univ. São Paulo; v. 9, n. 3, p. 94-104. 1998.

BEZERRA, K. V. Estudo do cotidiano e qualidade de vida de pessoas com insuficiência renal crônica (IRC), em hemodiálise. Dissertação (Mestrado em Saúde na Comunidade). Ribeirão Preto: Faculdade de Medicina de Ribeirão Preto da Universidade de São 
Paulo, 2006.

CARDOSO, A. A.; MAGALHÃES, L.C. Bilateral coordination and motor sequencing in Brazilian children: preliminary construct validity and reliability analysis. Occup. Ther. Int., v. 16, n. 2, p. 107-121, 2009.

CORDEIRO, J. R. Validade transcultural da lista de papéis ocupacionais para portadores de doença pulmonar obstrutiva crônica. Dissertação (Mestrado em Ciências Médicas). São Paulo: Universidade Federal de São Paulo, 2005.

GAMEIRO, C. M.; FERREIRA, I. Fiabilidade e sensibilidade do Loewenstein Occupational Therapy Cognitve Assesment (LOTCA). $\operatorname{Re}($ Habilitar) - Revista da ESSA, v. 2, p. 55-67. 2006.

KIELHOFNER, G. Model of human occupational: theory and application. Baltimore: Williams and Wilkins, 1985.

MAGALHÃES, L. C. Avaliação de terapia ocupacional: o quê avaliar e como avaliar. In: CONGRESSO BRASILEIRO, 5., SIMPÓSIO LATINO AMERICANO DE TERAPIA OCUPACIONAL, 4., Belo Horizonte, 1997. Horizontes da Clínica à Pesquisa. Anais. Belo Horizonte, MG, 1997.

MAGALHÃES, L. C.; NASCIMENTO, V. C. S.; REZENDE, M. B. Avaliação da coordenação e destreza motora - ACOORDEM: etapas de criação e perspectivas de validação. Rev. Ter Ocup. Univ. São Paulo, v. 15, n. 1, p. 17-25, 2004.

MENEZES, P. R.; NASCIMENTO, A. F. Validade e confiabilidade das escalas de avaliação em psiquiatria. In: GORENSTEIN,C., et al. Escalas de avaliação clínica em psiquiatria e psicofarmacologia.
Versão atualizada e ampliada. Rev. Psiquiatr. Clín., v. 25, n. 5,6, 1999; v. 26, n. 1,2, p. 24-28, 1998.

OLIVEIRA, A. S. Adequação e estudo de validade e fidedignidade da Escala Interativa de Observação de Pacientes Psiquiátricos Internados às Situações de Terapia Ocupacional. Dissertação (Mestrado em Saúde Mental). Ribeirão Preto: Universidade de São Paulo, 1995.

PESCE, R. P.; ASSIS, S. G.; AVANCI, J. Q.; SANTOS, N. C.;. MALAQUIAS, J.V.; CARVALHAES, R. Adaptação transcultural, confiabilidade e validade da escala de resiliência. Cad. Saúde Pública, v. 21, n. 2, p. 436-448. 2005.

TEDESCO, S. A. Estudo da validade e confiabilidade de um instrumento de Terapia Ocupacional: Auto-Avaliação do Funcionamento Ocupacional (SAOF). Dissertação (Mestrado em Saúde Mental). São Paulo: Escola Paulista de Medicina - Unifesp, 2000 .

TEDESCO, S. A. O desenvolvimento e estudo de instrumentos de avaliação em Terapia Ocupacional. Cad. Ter. Ocup. Centro Universitário S. Camilo, São Paulo, v. 8, n. 3, p. 17-26, 2002.

TEDESCO, S. A; CITER, V. A.; MARTINS, L. A. N.; IACOPONI, E. Tradução e validação para português brasileiro da Escala de Auto-avaliação do Funcionamento Ocupacional. Mundo Saúde, São Paulo, v. 34, n. 2, p. 230-237, 2010.

TSE, S.; PENMAN, M. From rhetoric to reality: Use of randomized controlled trials in evidence-based occupational therapy. Austr. Occup. Ther. J., v. 47, p. 181-185, 2000.

URBINA, S. Fundamentos da testagem psicológica. Tradução Claúdia Dornelles Porto Alegre: Artmed, 2007. p. 173-174. 